\title{
CORONARY ARTERY FISTULA
}

\author{
Anusha Sunkara, M.D.; Lakshmi H. Chebrolu, M.D.; Su Min Chang, M.D.; Colin Barker, M.D. \\ Houston Methodist Hospital, Houston, Texas
}

\begin{abstract}
Coronary artery fistulae are rare abnormalities of the coronary arteries that are usually discovered incidentally on coronary angiogram. Most patients remain asymptomatic, and significant hemodynamic consequences are rarely observed. The following report describes the case of a patient presenting with symptoms of congestive cardiac failure due to multiple coronary artery fistulae.
\end{abstract}

\section{Introduction}

Coronary artery fistula is a rare congenital or acquired condition that involves an abnormal communication between a coronary artery and either a cardiac chamber (coronary-cameral fistula) or any segment of systemic or pulmonary circulation (coronary-arteriovenous fistula). Although single-vessel coronary artery fistulae have frequently been reported in the literature, occurrence of multiple fistulae is rare. Here we present a unique case of a patient with multiple coronary artery fistulae with significant hemodynamic consequences.

\section{Case Presentation}

A 60-year-old female with a known history of gastric cancer presented with orthopnea and dyspnea on exertion (New York Heart Association class IV). On presentation she was afebrile with a heart rate of 102 beats per minute, respiratory rate of 20 breaths per minute, blood pressure of $113 / 53 \mathrm{~mm} \mathrm{Hg}$, and $\mathrm{O}_{2}$ saturation of $93 \%$ on 2 liters oxygen. Physical examination was significant for elevated jugular venous pressure $\left(15 \mathrm{~cm} \mathrm{H}_{2} \mathrm{O}\right)$, grade IV/VI systolic murmur best heard in the aortic area, and trace pedal edema. Transthoracic echocardiogram revealed a bicuspid aortic valve with mild to moderate stenosis (aortic valve area of $0.9 \mathrm{~cm}^{2}$ and mean aortic gradient of $22 \mathrm{~mm} \mathrm{Hg}$ ), normal left ventricular ejection fraction $(>60 \%)$, and significant pulmonary hypertension (50-55 mm Hg). A nuclear stress test was inconclusive and was followed by coronary angiography, which showed no significant obstructive disease. Incidental fistulae from the proximal right coronary artery and left anterior descending arteries were noted (Figures 1,2). A coronary computerized tomography angiogram (CTA) showed tortuous left main and left anterior descending coronary arteries with two large fistulae arising from the left anterior descending (LAD) (Figure 3). Another fistula from the right coronary artery (RCA) was also visualized (Figure 4). All the fistulae drained into the main pulmonary artery (PA). Percutaneous transcatheter embolization of the fistulae from the LAD to the main PA was attempted but was unsuccessful secondary to severe tortuosity of the vessel. The patient was followed closely and surgical ligation was considered, but she had a recurrence of metastatic gastric carcinoma and opted for comfort care and hospice.

\section{Discussion}

Coronary artery fistula is defined as an abnormal communication between the coronary arteries and a component of systemic or pulmonary circulation. ${ }^{1}$ The term "coronary-cameral fistula" refers to a communication between the coronary arteries and a cardiac chamber. ${ }^{1}$ Although most of them are congenital, acquired fistulae have been reported in cases of trauma, as a complication of coronary artery bypass surgery, and in some cases of Takayasu's arteritis. $^{2,3}$

The incidence of coronary artery fistulae is reported to be less than $1 \%$ in several studies. ${ }^{3-5}$ A review of the literature shows that there is no uniform consensus regarding the most common site of origin. However, the left main coronary artery is reported to be the most common site of origin in several studies, whereas the most common site of drainage is the pulmonary artery. ${ }^{3,6}$

The hemodynamic consequences depend on the size of the fistula, the pressure gradient along the fistula, and the volume of the shunt flow. ${ }^{1,3}$ Most of the fistulae are small and do not result in significant changes, although large fistulae with excessive flow rates draining into the right side of the heart can give rise to volume overload and pulmonary hypertension. Shunting of the blood through the fistula can also lead to "coronary steal syndrome" due to decreased blood flow to the myocardium distal to the fistula, thus leading to ischemia. ${ }^{1,3}$

Most patients are asymptomatic, and continuous cardiac murmur is the most commonly reported finding upon physical examination. ${ }^{7}$ The age at presentation in symptomatic patients varies significantly. Large fistulae with significant flow can present with signs and symptoms of congestive cardiac failure as early as in infancy. Angina, dyspnea on exertion, syncope, and palpitations are some of the other manifesting symptoms. ${ }^{1}$ In addition to volume overload and ischemia, other reported complications of coronary artery fistulae include arrhythmias, progressive enlargement, aneurysm and rupture, infective endocarditis, and sudden death. ${ }^{3}$

Most coronary artery fistulae are diagnosed incidentally during cardiac catheterization. Multidimensional CTA is gaining popularity as it is noninvasive and provides 3-dimensional visualization that helps to better delineate the anatomy of the coronary arteries. ${ }^{4}$ In fact, studies have shown that CTA detects coronary artery anomalies at a higher rate than traditional coronary angiography. ${ }^{4}$ Cardiac magnetic resonance imaging and 2-dimensional echocardiography are the other recognized modalities for diagnosing coronary artery fistulae. ${ }^{1,8,9}$

Therapeutic intervention with surgical ligation or transcatheter closure (TCC) is typically performed in patients with symptomatic fistula or in case of a complication. Percutaneous TCC is the preferred method as it is less invasive and avoids the potential complications of surgery. However, the anatomy may not be conducive to TCC, especially if the vessel is tortuous or in cases of a large fis- 


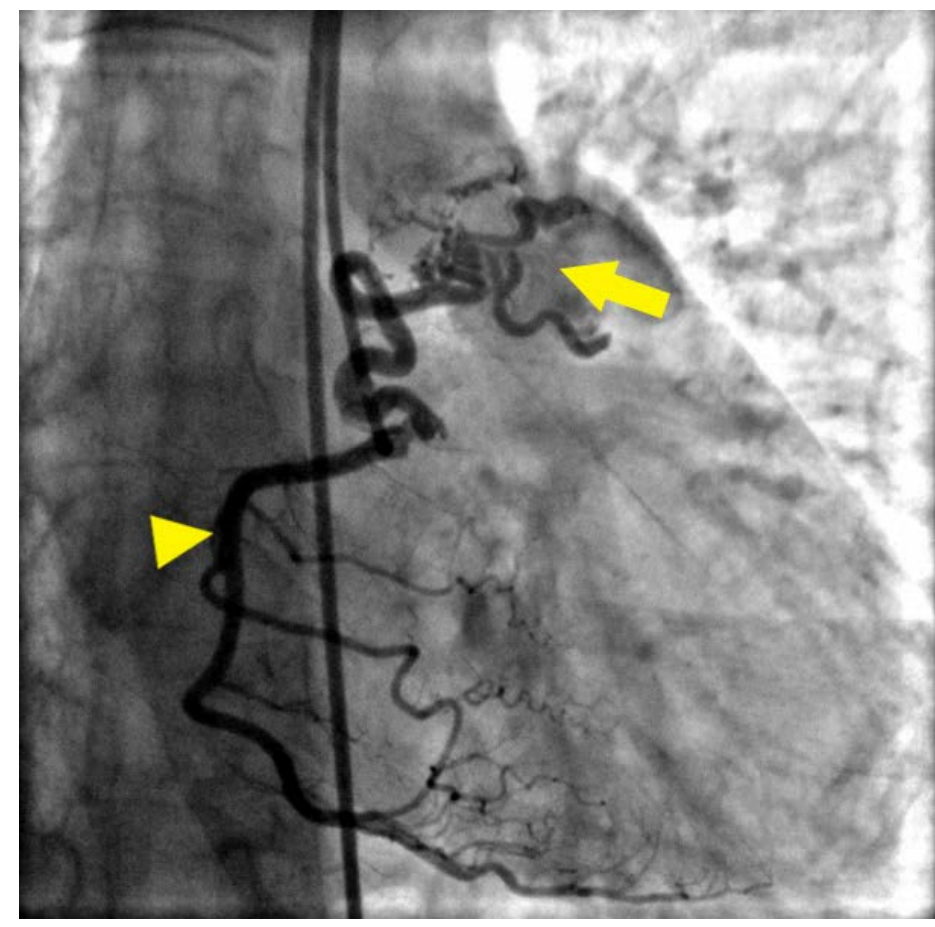

Figure 1. Coronary angiogram image showing fistula (arrow) from right coronary artery (arrowhead).

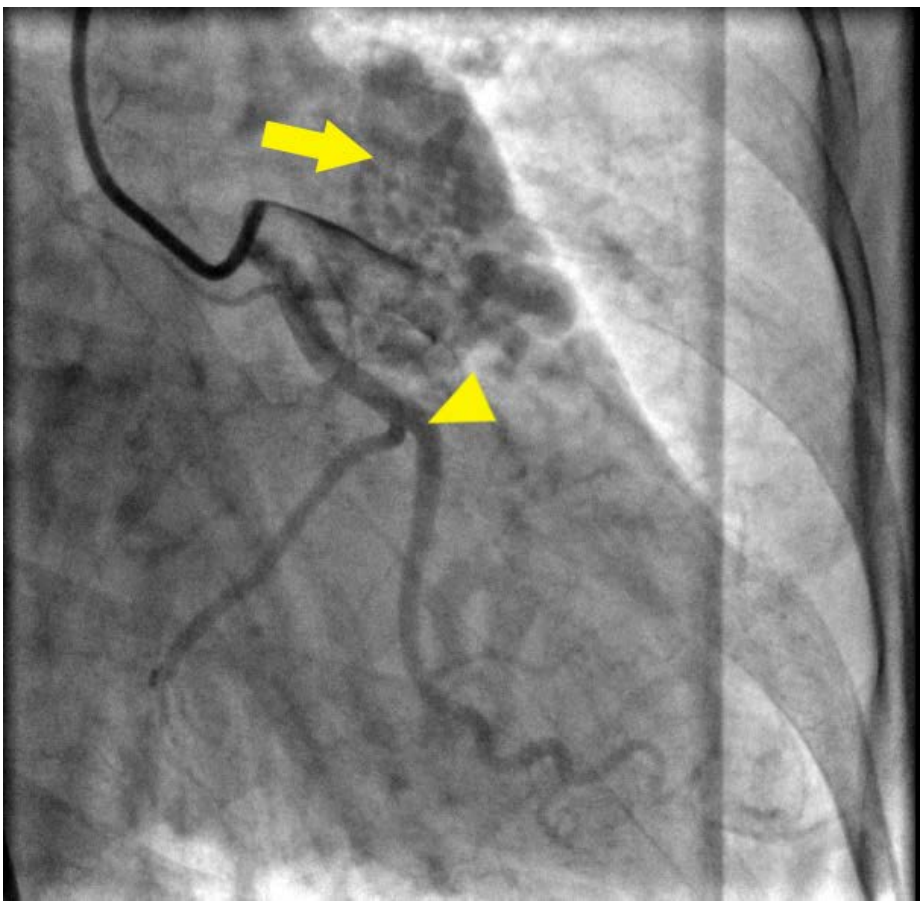

Figure 2. Coronary angiogram image showing fistula (arrow) from left anterior descending coronary artery (arrowhead).

tula; in these cases, surgical closure is preferred. ${ }^{1,10}$ There is no general consensus regarding the treatment in asymptomatic patients. Patients who have small fistulae with insignificant shunt can be managed with close follow-up. Elective closure of a fistula is recommended in children older than 3 to 5 years of age as they are at increased risk of complications. It is also recommended in cases of fistulae arising from the proximal segment of coronary arteries as they are prone to aneurysm formation and rupture. ${ }^{1}$

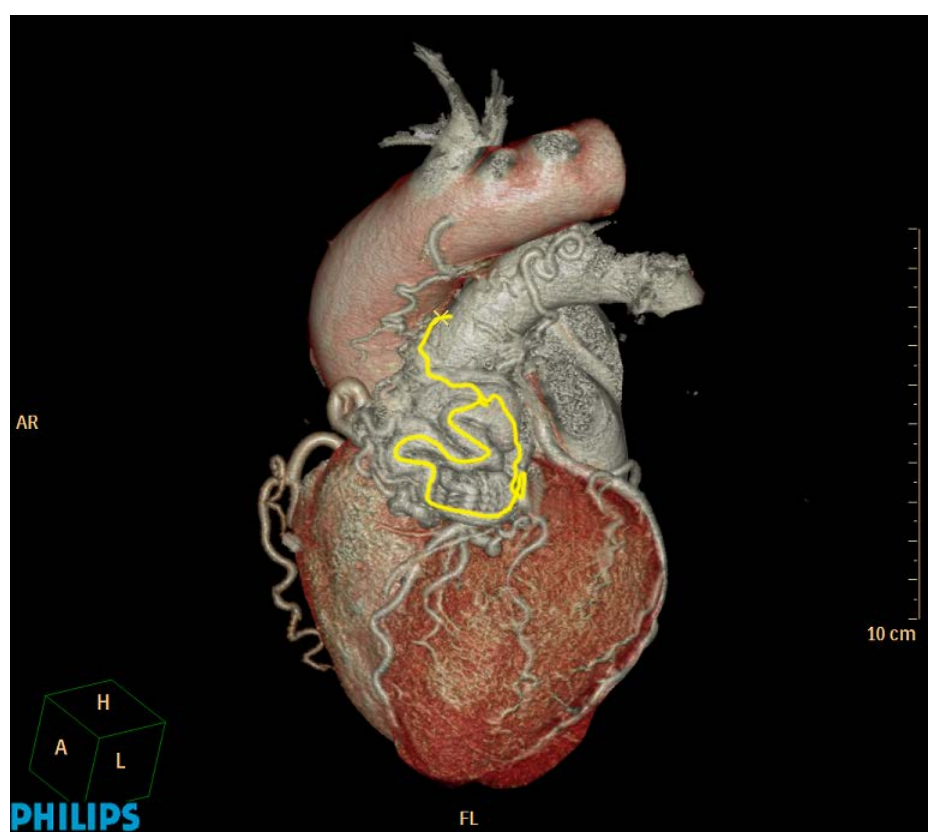

Figure 3. Coronary angiogram image showing fistula (yellow line) from left anterior descending coronary artery to pulmonary artery.

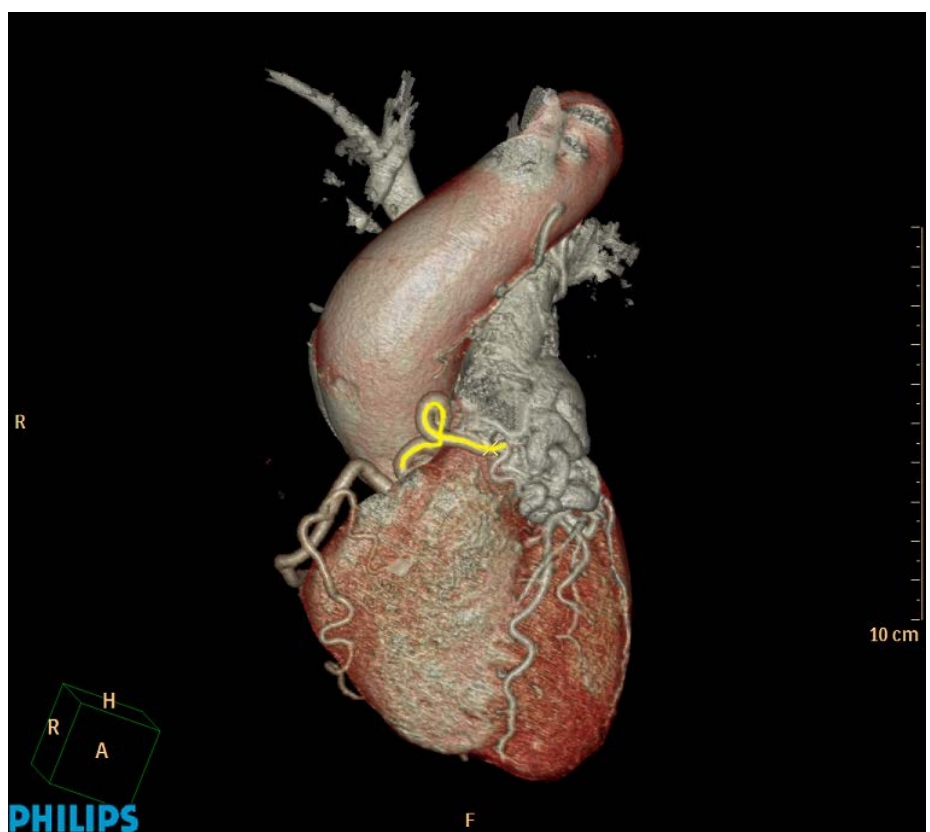

Figure 4. Coronary angiogram image showing fistula (yellow line) from right coronary artery to pulmonary artery.

\section{Summary}

In summary, coronary artery fistula is a rare congenital or acquired anomaly of the coronary artery. Although most patients are asymptomatic, large fistula with high flow rates can be symptomatic and can lead to life-threatening complications. Advanced imaging modalities such as CTA provide more accurate anatomy of the fistula compared to traditional coronary angiogram. Transcatheter closure of the fistula is the preferred modality of treatment in symptomatic patients. 
Conflict of Interest Disclosure: The authors have completed and submitted the Methodist DeBakey Cardiovascular Journal Conflict of Interest Statement and none were reported.

Keywords: coronary artery fistula, coronary-cameral fistula, computerized tomography angiogram

\section{References}

1. Challoumas D, Pericleous A, Dimitrakaki IA, Danelatos C, Dimitrakakis G. Coronary arteriovenous fistulae: a review. Int J Angiol. 2014 Mar;23(1):1-10.

2. Raufi MA, Baig AS. Coronary artery fistulae. Rev Cardiovasc Med. 2014 Jan;15(2):152-7.

3. Hobbs RE, Millit HD, Raghavan PV, Moodie DS, Sheldon WC. Coronary artery fistulae: a 10-year review. Cleve Clin Q. 1982 Winter;49(4):191-7.

4. Lim JJ, Jung Jl, Lee BY, Lee HG. Prevalence and types of coronary artery fistulas detected with coronary CT angiography. AJR Am J Roentgenol. 2014 Sep;203(3):W237-43.

5. Shabestari AA, Akhlaghpoor S, Tayebivaljozi R, Fattahi Masrour F. Prevalence of Congenital Coronary Artery Anomalies and Variants in 2697 Consecutive Patients Using 64-Detector Row Coronary CTAngiography. Iran J Radiol. 2012 Sep;9(3):111-21.

6. Kamiya $H$, Yasuda $T$, Nagamine $H$, et al. Surgical treatment of congenital coronary artery fistulas: 27 years' experience and a review of the literature. J Card Surg. 2002 Apr;17(2):173-7.

7. Said SA. Current characteristics of congenital coronary artery fistulas in adults: A decade of global experience. World J Cardiol. 2011 Aug 26;3(8):267-77.

8. Varghese A, Keegan J, Pennell DJ. Cardiovascular magnetic resonance of anomalous coronary arteries. Coron Artery Dis. 2005 Sep;16(6):355-64.

9. Velvis H, Schmidt KG, Silverman NH, Turley K. Diagnosis of coronary artery fistula by two-dimensional echocardiography, pulsed Doppler ultrasound and color flow imaging. J Am Coll Cardiol. 1989 Oct;14(4):968-76.

10. Armsby LR, Keane JF, Sherwood MC, Forbess JM, Perry SB, Lock JE. Management of coronary artery fistulae. Patient selection and results of transcatheter closure. J Am Coll Cardiol. 2002 Mar 20;39(6):1026-32. 